\title{
Recent Results from VERITAS AGN Observations
}

\section{Wystan Benbow ${ }^{a, *}$ on behalf of the VERITAS Collaboration}

(a complete list of authors can be found at the end of the proceedings)

${ }^{a}$ Center for Astrophysics | Harvard \& Smithsonian,

60 Garden St, Cambridge, MA, 02138, USA

E-mail: wbenbow@cfa.harvard.edu

VERITAS is one of the world's most sensitive detectors of astrophysical very high energy (VHE; $\mathrm{E}>100 \mathrm{GeV}$ ) gamma rays. This observatory has operated for $\sim 14$ years, and nearly 7,000 hours of its observations have been targeted on active galactic nuclei (AGN). Approximately 300 AGN were observed with VERITAS, and 40 are detected. These studies are generally accompanied by contemporaneous, broadband observations, which enable detailed probes of the underlying jet-powered processes. Recent scientific results from VERITAS AGN observations are presented.

$37^{\text {th }}$ International Cosmic Ray Conference (ICRC 2021)

July 12th-23rd, 2021

Online - Berlin, Germany

\footnotetext{
${ }^{*}$ Presenter
} 


\section{Introduction}

AGN comprise $\sim 34 \%$ of the VHE sky catalog and are the most numerous class of identified VHE $\gamma$-ray source [1]. These objects have long dominated the observing programs of Northern VHE observatories such as VERITAS [2]. As of ICRC 2021, AGN comprise approximately $52 \%$ of the known VHE sources with Northern declinations $\left(\delta>0^{\circ}\right)$, and $76 \%$ of the 84 VHEdetected AGN are located in the North. These dominantly non-thermal sources have spectral energy distributions (SEDs) spanning from radio waves through $\gamma$-rays. Their emission is highly variable at all wavelengths, and on time scales as short as minutes. This leads to an emphasis on using contemporaneous multi-wavelength (MWL) observation campaigns to probe their underlying physics. It is generally agreed that the VHE $\gamma$-ray emission from AGN is produced in their relativistic jet, in a compact region near their central, supermassive black hole. However, some recent detections show indications for VHE emission produced on larger and/or more distant scales (see, e.g., [3]). The characteristic double-peaked SEDs observed from AGN naturally suggest emission models where the low-energy peak is the synchrotron radiation from a population of relativistic electrons in the relativistic jet, and the high-energy photons are the products of inverse-Compton scattering from this population (e.g. a synchrotron self-Compton (SSC) model as in [9]). While VHE emission models dominated by leptonic particle-acceleration processes in the accretion-powered jets remain favored, other models remain viable (see, e.g., [16] and the discussion / references therein).

Blazars are AGN whose relativisitc jets are pointed along the line of sight towards Earth. They form the dominant class ( 95\%) of VHE AGN. Of the 78 VHE blazars, 67 ( $\sim 90 \%)$ are BL Lac objects, 8 are Flat Spectrum Radio Quasars (FSRQs) and 3 have uncertain sub-classification. Of the remaining 6 VHE AGN, at least four are nearby $(z<0.022)$ FR-I radio galaxies, and the radiogalaxy / blazar classification for another 2 remains debated. In any case, the jets for these 6 objects are not strongly misaligned. The sub-classification of BL Lac objects, based on the location of their lower-energy SED peak, is important. Within the VHE catalog, 53 BL Lacs are high-frequencypeaked (HBLs), 10 are intermediate-frequency-peaked (IBLs), 2 are low-frequency-peaked (LBLs) and two are unclassified. The VHE blazar catalog currently covers a redshift range from $z=0.030$ to $z=0.954$, but at least $\sim 20 \%$ of the objects have unknown redshift and many catalog values are uncertain. This is in large part due to the absence of optical features in the spectra of BL Lac objects. Generally, the VHE AGN catalog is peaked at nearby redshifts (e.g., $~ 55 \%$ have $z<0.2$ ) but $\sim 10 \%$ of the VHE objects have $z>0.5$ (primarily FSRQs). Aside from energetics considerations, the major contributor to this redshift distribution is attenuation of VHE photons in a distance- and energy-dependent manner by the extragalactic background light (EBL) [6].

In general, the observational goals of the VERITAS AGN (radio galaxy and blazar) Program are to discover new VHE AGN, and to make precision measurements of VHE AGN spectra and their variability patterns. There are two empirical qualities of the AGN population that drive the strategy behind the VERITAS program. The first is that most VHE AGN have shown at least some VHE flux variability. Indeed major outbursts are perhaps one of the defining characteristics of the VHE field (see, e.g., [7]), and roughly one-third of VHE AGN are only detected during brief flares. These flare-only AGN include most of the non-HBL blazars. While extreme episodes may define the field, these rapid (minute-scale), large-scale (factor of 100) flux variations are very rare. Most VHE flux variations are mild (factor of 2-3) and many have comparatively long time scales (e.g., 


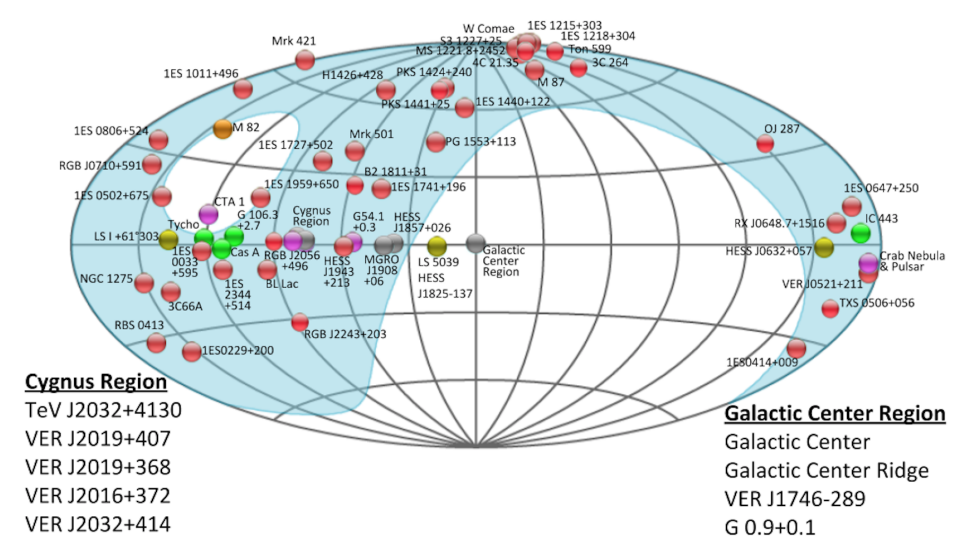

Figure 1: The VERITAS catalog in Galactic coordinates. The red circles show the 40 AGN detected using VERITAS; other astrophysical classes are shown with different colors. The blue region is visible to VERITAS at zenith angles $<35^{\circ}$. Most of the VERITAS AGN catalog is given in [15]; only the new IBL B2 1811+31 (see below) is missing.

an observing season). In general the time-scale and strength of the variations observed depends on the average VHE flux, but this may have biases (e.g., instrument sensitivity, target selection, etc.) The VERITAS AGN Program therefore attempts to identify and follow-up on VHE AGN flares, guided in part by brighter than average VHE flux. The other important empirical quality is that the observed photon spectra of VHE AGN are often soft $\left(\Gamma_{\text {avg }} \sim 3.5\right)$, and very few VHE AGN are detected above $1 \mathrm{TeV}$. This partly due to the high-energy SED peak location, which may depend on the underlying physics, and partly due to propagation effects (e.g., EBL absorption). AGN with hard VHE spectra are particularly interesting, and the VERITAS AGN Program also focuses on hard spectrum VHE blazars and generating statistics above above $1 \mathrm{TeV}$. A study of the spectra measured by VERITAS from these VHE blazars can be found in these proceedings [19].

The VERITAS AGN Program strongly leverages contemporaneous MWL observations from numerous ground- and space-based facilities. Swift XRT/UVOT, Fermi-LAT, the FLWO 48" optical telescope form the core of this regular lower-energy coverage, which exists for all Northern VHE AGN. After an initial focus on expanding the VHE AGN catalog from 2007-10, the VERITAS AGN program has since emphasized studies of the known VHE AGN population with a goal of identifying and intensely observing major flares. The target list has evolved but the general method was to sample each selected VHE AGN on a regular cadence, thereby building high-statistics data sets while searching for flares. VERITAS AGN observations are timed to the MWL coverage to enable fully-constrained modeling of each VHE AGN's SED (see, e.g., [9]). From 2013-2018, the program sampled each Northern VHE AGN ( $\sim 56$ targets), but it was recently streamlined to focus on more intense studies of select $(\sim 23)$ targets. Our current focus is on the most variable VHE sources (i.e. IBLs), bright HBLs, and hard-spectrum HBLs. This program has yielded detailed, decade-long MWL light curves for numerous ( 20) sources and significant coverage for the entire Northern population, in addition to the identification of numerous VHE flares. The ongoing sampling continues to improve these efforts, and the various long-term MWL light curves will enable flux and spectral correlation studies that may indicate commonalities in the origin of each AGN's emission. The large ensemble of precision VHE AGN spectra will also improve and has already proved useful for generating a variety of cosmological measurements such as constraints on the the density of the EBL [6] and the strength of the intergalactic magnetic field (IGMF) [10]. 


\section{VERITAS AGN Program}

VERITAS began full-scale operations in 2007 at the F. L. Whipple Observatory in Arizona, USA. After fourteen years, it remains the world's most sensitive observatory between $\sim 85 \mathrm{GeV}$ and $\sim 30 \mathrm{TeV}$. VERITAS scientists use the array of four Cherenkov telescopes to study the Northern sky during $\sim 10$-month, monsoon-limited seasons (September - July). The present sensitivity was achieved following a series of upgrades in 2012, and VERITAS can detect an object with $\sim 1 \%$ Crab Nebula flux (1\% Crab) in less than 25 hours. Photon spectra can be generated above $\sim 100 \mathrm{GeV}$ with typical systematic errors of $\sim 0.1$ on the photon index $(\Gamma)$ and $\sim 20 \%$ on the flux.

The VERITAS collaboration has acquired $\sim 16,300 \mathrm{~h}$ of observations. While successful, the past two seasons were challenging due to the global pandemic. The 2019-20 season ended $\sim 4$ months early causing an estimated loss of $\sim 500 \mathrm{~h}$ of data ( $\sim 700 \mathrm{~h}$ were acquired). A rapid shift to "remote" operations largely enabled a full-scale observing program in 2020-21, but it required pausing the bright-moon observing program $(\sim 1000 \mathrm{~h}$ acquired; only $\sim 40 \mathrm{~h}$ bright-moon data); this will resume in Fall 2021. Excluding the pandemic-affected seasons, VERITAS averages $\sim 930 \mathrm{~h} /$ season of good-weather observations during "dark time" (moon illumination $<30 \%$ ), and $\sim 200 \mathrm{~h} /$ season during periods of "bright moonlight" (i.e. $>30 \%$ illumination). The bright-moon data has similar sensitivity to dark-time observations, but has higher threshold (e.g. $250 \mathrm{GeV}$ ) [11].

AGN comprise $63 \%$ of the VERITAS source catalog (shown in Figure 1), and their observations are the dominant component of VERITAS data taking ( $~ 50 \%)$. As of July 2021, AGN data comprise a total of $\sim 5,900 \mathrm{~h}(\sim 420 \mathrm{~h}$ per year) of good-weather dark time, $\sim 1,100 \mathrm{~h}(\sim 140 \mathrm{~h}$ per year $)$ of good-weather, bright-moon time, and $\sim 1,800 \mathrm{~h}$ poor-weather (filler) observations. Blazars are the dominant component of the AGN program, and the good-weather dark time is historically split $\sim 90 \%$ to blazars, primarily BL Lac objects, and $\sim 10 \%$ to radio-galaxies. From 2019-21, VERITAS acquired $\sim 670 \mathrm{~h}$ of good-weather dark time on blazars and $\sim 100 \mathrm{~h}$ of good-weather dark time on radio galaxies, showing a larger emphasis on radio galaxies. There were a further $\sim 90 \mathrm{~h}$ of goodweather, bright-moon time acquired on blazars. After initially using 35-45\% of the bright-moon time for blazar discoveries, almost all of this time ( $90 \%$ from 2019-21) is now used for observing hard-spectrum BL Lac objects and searching for VHE AGN flares from targets not in the regular VERITAS AGN monitoring program. The filler data is also used to search for AGN flares. These supplemental flare monitoring programs have found several events not otherwise identified.

Much of the AGN observing program is based on regular monitoring of selected objects in the Northern VHE catalog. The depth and cadence of these observations is based on a variety of criteria and scientific goals. These exposures aim to both to self-identify VHE flaring episodes for immediate / intense target-of-opportunity (ToO) follow-up including MWL partners, while simultaneously building deeper, legacy exposures on particularly interesting objects. Figure 2 shows the distribution of exposures already achieved by VERITAS for each Northern AGN. The monitoring cadence ranges from daily to weekly, while the minimum sample duration will detect $\sim 10 \%$ Crab flux. While the general goal of the program is to identify long-lasting, flare states for intense campaigns, our experience shows that we are also able to fortuitously catch short-duration, bright flares (e.g. BL Lac in 2017 [4]). Contemporaneous MWL data (e.g. Swift) are timed with the VERITAS monitoring observations to both assist with triggering and to enable long-term modeling. For the 2020-21 season, VERITAS monitored 23 VHE AGN (37\% of the Northern population). 

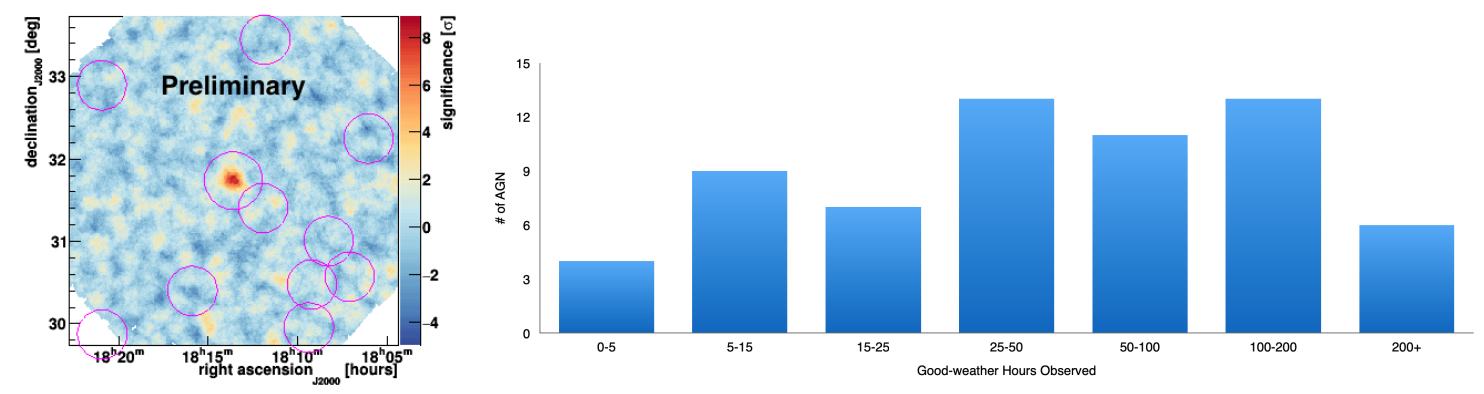

Figure 2: (Left) The preliminary sky map of the significance measured from the direction of B2 1811+31. (Right) Histogram of the total VERITAS exposure for every Northern VHE AGN; $\sim 50 \%$ already have more than $50 \mathrm{~h}$.

While a major focus of the AGN program is performing deep / timely measurements of known VHE sources, $\sim 35 \%$ of the 2019-21 data were devoted to the discovery of new VHE AGN. This included regular observations of targets from a list of selected candidates, and ToO observations triggered by our partners. Our discovery candidates include several AGN with a weak excess $(>3 \sigma)$ in large, archival VERITAS exposures; these excesses continue to grow in limited annual exposures. In addition, we continue a program to observe all targets from a comprehensive list of Northern discovery candidates for at least $5 \mathrm{~h}$. Only 11 objects require further observation, and most have at least some exposure. The target list includes all the X-ray bright HBLs in the 2WHSP catalog ("TeV Figure of Merit" > 1.0; [17]), all the hardest AGN in the Fermi-LAT $>50 \mathrm{GeV}$ catalog $\left(\Gamma_{2 F H L}<2.8 ;\right.$ [8], all nearby $(z<0.3)$ LBLs from the MOJAVE sample with relatively high maximum apparent jet speed [21], and all targets from prior comprehensive efforts [13, 14].

ToO observations are the highest priority of the VERITAS program. These data comprised $12 \%$ and $15 \%$ of the blazar dark-time in the 2019-20 and 2020-21 seasons, respectively. Approximately $70 \%$ of these data were follow-up observations of flares in known VHE blazars, with the remainder including attempts to discover and/or follow-up on new VHE blazars.

\section{Recent Highlights}

B2 1811+31 is an IBL at redshift $z=0.117$ that showed an elevated MeV-GeV flux $(\sim 11 \mathrm{x}$ brighter) and harder gamma-ray spectrum $\left(\Gamma_{\mathrm{LAT}} \sim 1.4\right.$ vs. 2.1$)$ during Fermi-LAT observations in Oct. 2020 (ATel \#14060). Following the discovery of VHE emission by MAGIC (Oct. 4-10; ATel $\# 14090$ ), and reports of enhanced optical activity (ATel \#14103), VERITAS observed the blazar from Oct. 15-19, 2020. A preliminary analysis of these data $(\sim 5 \mathrm{~h})$ yields a strong detection $(\sim 8$ standard deviations, $\sigma)$ and a soft photon spectrum $(\Gamma=4.1 \pm 0.5)$. A sky map of the significance observed near B2 $1811+31$ is shown in Figure 2. The VERITAS light curve is consistent with a constant flux $\mathrm{F}(>250 \mathrm{GeV})=\left(1.10 \pm 0.18_{\text {stat }}\right) \times 10^{-11} \mathrm{~cm}^{-2} \mathrm{~s}^{-1}$. This is approximately $6 \%$ of the Crab Nebula flux (Crab) above the same threshold, and is similar to the flux reported by MAGIC.

$\mathbf{H ~ 1 4 2 6 + 4 2 8}(z=0.129)$ is an extreme HBL with synchrotron peak located at $10^{18.1} \mathrm{~Hz}$ [17]. It was routinely detected before 2002 with VHE fluxes between $\sim 5 \%$ and $\sim 20 \%$ Crab. However, since this time the reported VHE fluxes are significantly less ( $<2-3 \%$ Crab). H 1426+428 was observed with VERITAS in almost every season and a $\sim 200 \mathrm{~h}$ exposure exists. A preliminary analysis of $\sim 82$ $\mathrm{h}$ of good-quality data from 2008-2016 yields a $13 \sigma$ detection. Although MWL observations show 

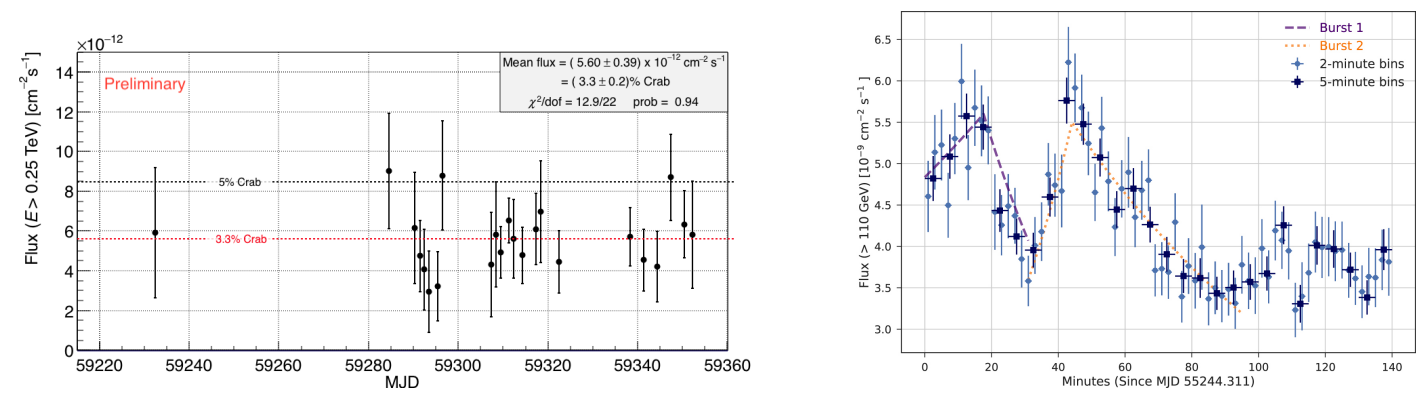

Figure 3: (Left) The preliminary nightly light curve from VERITAS observations of H 1426+428 in 2021. (Right) The VERITAS light curve from Mrk 421 on Feb. 17, 2010 [7]. The dashed lines are exponential fits to the two bursts (2-min-bins). The resulting timescales are 84 and $22 \mathrm{~min}$ for doubling (rising), and 28 and 65 min for halving (decay).

variations (e.g., the Swift XRT count rate varies by a factor of 3), the VHE flux $\mathrm{F}(>250 \mathrm{GeV})=$ $(1.9 \pm 0.2) \%$ Crab shows no signs of variability. The preliminary VHE spectrum can be described by a power law with $\Gamma=2.8 \pm 0.1$. During 2021 monitoring observations, $\mathrm{H} 1426+428$ was found to be in a bright VHE state. This triggered an intense VERITAS and MWL campaign including Swift and NuStar. Overall, $\sim 45 \mathrm{~h}$ of VERITAS data were acquired. A preliminary analysis of these data yields a $\sim 19 \sigma$ detection and a time-average spectrum $(\Gamma \sim 2.6)$ consistent with the long-term measurement. The preliminary flux, $\mathrm{F}(>250 \mathrm{GeV})=\left(5.6 \pm 0.4_{\text {stat }}\right) \times 10^{-12} \mathrm{~cm}^{-2} \mathrm{~s}^{-1}$, or $(3.3 \pm$ $0.2) \% \mathrm{Crab}$, is steady throughout 2021 but higher than the 2008-16 average. The preliminary light curve from the 2021 observations is shown in Figure 3. The steady VERITAS flux contrasts with the Swift-XRT monitoring which indicates a high count rate and significant variability.

3C 264 is an FR-I type radio galaxy at $z=0.0216$. It was observed with VERITAS for $57 \mathrm{hr}$ from 2017-19, resulting in the 7.8 $\sigma$ discovery of VHE emission [12]. Its VHE flux is variable on monthly timescales and was elevated in 2018. The hard-spectrum $(\Gamma \sim 2.20)$ VHE emission during 2018 has $\mathrm{F}(>315 \mathrm{GeV})=\left(7.6 \pm 1.2_{\text {stat }} \pm 2.3_{\text {syst }}\right) \times 10^{-13} \mathrm{~cm}^{-2} \mathrm{~s}^{-1}(0.7 \% \mathrm{Crab})$. The elevated VHE state was thought to be similar to those observed from the analogous source, M 87, and extensive contemporaneous MWL data were acquired in 2018, including high-resolution imaging data (e.g., Chandra, HST, VLA, VLBA). However, there was no clearly identifiable source of the elevated flux. The 3C 264 SED is unusual for a radio galaxy; its synchrotron peak near the X-rays has relatively high frequency. However, many aspects of the SED can be qualitatively reproduced with an SSC model using parameters typical of BL Lacs. Comparing the SEDs of 3C 264 and M 87 shows differences that are plausibly explained by 3C 264 being oriented closer to the line of sight.

Mrk 421 exhibited an extraordinary flaring episode in February 2010 [7]. The VHE flux observed by VERITAS from this HBL during an intense MWL campaign reached $27 \mathrm{Crab}$, the highest ever observed from an AGN. The light-curve from the brightest night is shown in Figure 3 and enables detailed cross-correlation analyses. Limits on the Doppler factor $(\delta \gtrsim 33)$ and the size of the emission region $\left(R_{B} / \delta \lesssim 3.8 \times 10^{13} \mathrm{~cm}\right)$ are obtained from the fast variability. A lag $(25-55$ minutes) between the VHE and optical bands is seen $(3 \sigma)$ for the first time on short time scales. The VHE and X-ray fluxes show a wide range of behavior, including linear and quadratic correlations, as well as anti-correlations. The MWL data are difficult to explain using a single-zone SSC model.

TXS 0506+056 is an important object for multi-messenger astronomy (see [5] and references therein). It was observed to have an elevated VHE and MeV-GeV gamma-ray flux in spatial and 
temporal coincidence $(\sim 3 \sigma)$ with the IceCube high energy neutrino event IC170922A. This could indicate that blazar jets accelerate cosmic rays to at least several PeV, and are hence a source of VHE cosmic rays. The association was also used to herald the birth of neutrino astronomy. The initial VERITAS follow-up observations of the neutrino/blazar ( $\sim 35 \mathrm{~h}$ from Sept. 2017 to Feb. 2018) led to a soft-spectrum $(\Gamma \sim 4.8)$, VHE detection $(5.8 \sigma)$, albeit at lower VHE flux than detected by MAGIC. VERITAS has since carried out deep observations of TXS 0506+056 and an associated MWL campaign [20]. A weak excess $(3.4 \sigma)$ is observed in $\sim 61 \mathrm{~h}$ collected from Oct. 2018 to Feb. 2021. Interpreting this excess as a detection, it corresponds to $\mathrm{F}(>190 \mathrm{GeV}) \sim 0.5 \% \mathrm{Crab}$. This is slightly lower than, but statistically consistent with, the prior VERITAS flux $(\sim 0.7 \% \mathrm{Crab})$.

The TeV luminosity function of HBLs is important because these AGN dominate the extragalactic VHE sky and hence the total cosmic VHE radiation. Its measurement (i.e. the number of HBLs per unit volume per unit luminosity) is key to understanding HBL properties, their relationship with other sources, and their contributions to unresolved radiation fields. This measurement is challenging due to observational biases, but enables studies of hadronic/neutrino production in jets, the IGMF, and AGN evolution. A program [18] was designed to minimize these biases by selecting 36 HBLs from the 3 WHSP catalog, and measuring their VHE fluxes at times not weighted towards high-fluxes. These VERITAS observations are complete and leverage $\sim 1800 \mathrm{~h}$ of archival data and $\sim 150 \mathrm{~h}$ of 2019-21 data. Each target has at least $8 \mathrm{~h}$ of exposure ( $\sim 1 \%$ Crab sensitivity).

FSRQs: are generally detected at VHE energies during flaring states. VERITAS has detected 3 FRSQs and most of its FSRQ observations are ToO based. In 2020, VERITAS began the first systematic search for VHE emission from FSRQs. Twelve objects were selected for at least 8 hours of unbiased (non-ToO) observations based on the 3FHL catalog and/or prior VHE detection. The data provide a sensitivity to fluxes of $\sim 1 \%$ Crab. Upper limits from the first 4 FSRQ (GB6 J0043+3426, S3 0218+35, PKS 0736+17 and 3C 454.3) are described in [22]. When complete, the survey will provide the first constraints on the duty cycle of VHE emission from FSRQs.

\section{Conclusion}

As of July 2021, the VERITAS collaboration has acquired 7,000 good-weather hours targeted on AGN. Since ICRC 2019, the array was used to acquire $\sim 860 \mathrm{~h}$ of these observations. Unfortunately, each of the past two seasons were affected by the global pandemic, with yields reduced by a 4-month observatory closure in 2019-20, and a temporary suspension of bright-moon observing in 2020-21. Overall, the 2019-21 AGN yield was $~ 25 \%$ below the two-year average.

The VERITAS AGN program continued to focus on deep, regular VHE and MWL monitoring of known VHE AGN, and immediate and intense ToO follow-up of interesting flaring events. We also maintained a robust discovery program, with $\sim 35 \%$ of our most recent observations having a discovery focus. Highlights from our recent AGN observations and publications include the observation of flares from B2 1811+31, H 1426+428, 3C 264 and Mrk 421.

The VERITAS array is now $\sim 14$ years old and continues to run well. Indeed, the past four seasons each rank among the five best for various technical performance benchmarks (e.g., fraction of data with all telescopes operational). The collaboration plans to operate VERITAS through at least 2025, and will continue prioritizing AGN observations. Given the array's strong technical performance, we expect the long VERITAS tradition of producing exciting AGN results to continue. 
This research is supported by grants from the U.S. Department of Energy Office of Science, the U.S. National Science Foundation and the Smithsonian Institution, by NSERC in Canada, and by the Helmholtz Association in Germany. This research used resources provided by the Open Science Grid, which is supported by the National Science Foundation and the U.S. Department of Energy's Office of Science, and resources of the National Energy Research Scientific Computing Center (NERSC), a U.S. Department of Energy Office of Science User Facility operated under Contract No. DE-AC02-05CH11231. We acknowledge the excellent work of the technical support staff at the Fred Lawrence Whipple Observatory and at the collaborating institutions in the construction and operation of the instrument.

\section{References}

[1] http://tevcat.uchicago.edu/

[2] https://veritas.sao.arizona.edu/specifications

[3] A.U. Abeysekara et al., "Gamma-Rays from the Quasar PKS 1441+25: Story of an Escape", ApJ, 815, L22, 2015.

[4] A. Abeysekara et al., "Multiwavelength Observations of the Blazar BL Lacertae: A New Fast TeV Gamma-Ray Flare", ApJ, 856, 95, 2018.

[5] A. Abeysekara et al., "VERITAS Observations of the BL Lac Object TXS 0506+056", ApJ, 861, L20, 2018.

[6] A.U. Abeysekara et al., "Measurement of the Extragalactic Background Light Spectral Energy Distribution with VERITAS", ApJ, 885, 150, 2019.

[7] A.U. Abeysekara et al., "The Great Markarian 421 Flare of 2010 February: Multiwavelength Variability and Correlation Studies", ApJ, 890, 97, 2020.

[8] M. Ackermann et al., "2FHL: The Second Catalog of Hard Fermi-LAT Sources", ApJS, 222, 1, 2016.

[9] E. Aliu et al., "A Three-Year MWL Study of the VHE $\gamma$-ray Blazar 1ES 0229+200", ApJ, 782, 13, 2014.

[10] S. Archambault et al., "Search for Magnetically Broadened Cascade Emission From Blazars with VERITAS", ApJ, 835, 288, 2017.

[11] S. Archambault et al., "Gamma-ray Observations Under Bright Moonlight with VERITAS", Astroparticle Physics, 91, 34, 2017.

[12] A. Archer et al., "VERITAS Discovery of VHE Emission from the Radio Galaxy 3C 264: A Multiwavelength Study", ApJ, 896, 14, 2020.

[13] W. Benbow, "The VERITAS Blazar Key Science Project", Proc. of the 31st ICRC (Lodz), 2009; arXiv:0908.1412.

[14] W. Benbow, "VHE Blazar Discoveries with VERITAS", Proc. of the 32nd ICRC (Beijing), 2011; arXiv:1110.0040.

[15] W. Benbow, "Highlights from the VERITAS AGN Observation Program", Proc. of the 36th ICRC (Madison), 2019; arXiv:1909.05323.

[16] M. Cerruti, M., W. Benbow, et al., "Luminous and high-frequency peaked blazars: the origin of the $\gamma$-ray emission from PKS 1424+240", A\&A, 606, 68, 2017.

[17] Y.-L. Chang et al., "2WHSP: A multi-frequency selected catalogue of high energy and very high energy $\gamma$-ray blazars and blazar candidates", $A \& A, \mathbf{5 9 8}, 17,2017$.

[18] M. Errando, "The luminosity function of TeV-emitting BL Lacs: observations of an HBL sample with VERITAS", Proc. of the 37th ICRC (Berlin), 2021.

[19] Q. Feng, "Exploring the high-energy gamma-ray spectra of TeV blazars", Proc. of the 37th ICRC (Berlin), 2021.

[20] W. Jin, "VERITAS follow-up observation of the blazar TXS 0506+056", Proc. of the 37th ICRC (Berlin), 2021.

[21] M. Lister, "Parsec-Scale Studies of Blazar Jet Kinematics", Half a Century of Blazars \& Beyond (Turin)", Presentation, 2018; https://agenda.infn.it/event/14538/contributions/25407/attachments/18101/20509/Lister.pdf

[22] S. Patel, "TeV emission from FSRQs: The first systematic \& unbiased survey", Proc. of the 37th ICRC (Berlin), 2021. 


\section{Full Authors List: VERITAS Collaboration}

C. B. Adams ${ }^{1}$, A. Archer ${ }^{2}$, W. Benbow ${ }^{3}$, A. Brill ${ }^{1}$, J. H. Buckley ${ }^{4}$, M. Capasso ${ }^{5}$, J. L. Christiansen ${ }^{6}$, A. J. Chromey ${ }^{7}$, M. Errando ${ }^{4}$, A. Falcone ${ }^{8}$, K. A. Farrel1 ${ }^{9}$, Q. Feng ${ }^{5}$, G. M. Foote ${ }^{10}$, L. Fortson ${ }^{11}$, A. Furniss ${ }^{12}$, A. Gent ${ }^{13}$, G. H. Gillanders ${ }^{14}$, C. Giuri ${ }^{15}$, O. Gueta ${ }^{15}$, D. Hanna ${ }^{16}$, O. Hervet ${ }^{17}$, J. Holder ${ }^{10}$, B. Hona ${ }^{18}$, T. B. Humensky ${ }^{1}$, W. Jin ${ }^{19}$, P. Kaaret ${ }^{20}$, M. Kertzman ${ }^{2}$, T. K. Kleiner ${ }^{15}$, S. Kumar ${ }^{16}$, M. J. Lang ${ }^{14}$, M. Lundy ${ }^{16}$, G. Maier ${ }^{15}$, C. E McGrath ${ }^{9}$, P. Moriarty ${ }^{14}$, R. Mukherjee ${ }^{5}$, D. Nieto ${ }^{21}$, M. Nievas-Rosillo ${ }^{15}$, S. O’Brien ${ }^{16}$, R. A. Ong ${ }^{22}$, A. N. Otte ${ }^{13}$, S. R. Pate ${ }^{15}$, S. Patel ${ }^{20}$, K. Pfrang ${ }^{15}$, M. Pohl $1^{23,15}$, R. R. Prado ${ }^{15}$, E. Pueschel ${ }^{15}$, J. Quinn ${ }^{9}$, K. Ragan ${ }^{16}$, P. T. Reynolds ${ }^{24}$, D. Ribeiro ${ }^{1}$, E. Roache ${ }^{3}$, J. L. Ryan ${ }^{22}$, I. Sadeh ${ }^{15}$, M. Santander ${ }^{19}$, G. H. Sembroski ${ }^{25}$, R. Shang ${ }^{22}$, D. Tak ${ }^{15}$, V. V. Vassiliev ${ }^{22}$, A. Weinstein ${ }^{7}$, D. A. Williams ${ }^{17}$, and T. J. Williamson ${ }^{10}$

${ }^{1}$ Physics Department, Columbia University, New York, NY 10027, USA ${ }^{2}$ Department of Physics and Astronomy, DePauw University, Greencastle, IN 46135-0037, USA ${ }^{3}$ Center for Astrophysics | Harvard \& Smithsonian, Cambridge, MA 02138, USA ${ }^{4}$ Department of Physics, Washington University, St. Louis, MO 63130, USA ${ }^{5}$ Department of Physics and Astronomy, Barnard College, Columbia University, NY 10027, USA ${ }^{6}$ Physics Department, California Polytechnic State University, San Luis Obispo, CA 94307, USA ${ }^{7}$ Department of Physics and Astronomy, Iowa State University, Ames, IA 50011, USA ${ }^{8}$ Department of Astronomy and Astrophysics, 525 Davey Lab, Pennsylvania State University, University Park, PA 16802, USA ${ }^{9}$ School of Physics, University College Dublin, Belfield, Dublin 4, Ireland ${ }^{1}$ ODepartment of Physics and Astronomy and the Bartol Research Institute, University of Delaware, Newark, DE 19716, USA ${ }^{11}$ School of Physics and Astronomy, University of Minnesota, Minneapolis, MN 55455, USA ${ }^{12}$ Department of Physics, California State University - East Bay, Hayward, CA 94542, USA ${ }^{13}$ School of Physics and Center for Relativistic Astrophysics, Georgia Institute of Technology, 837 State Street NW, Atlanta, GA 30332-0430 ${ }^{14}$ School of Physics, National University of Ireland Galway, University Road, Galway, Ireland ${ }^{15}$ DESY, Platanenallee 6, 15738 Zeuthen, Germany ${ }^{16}$ Physics Department, McGill University, Montreal, QC H3A 2T8, Canada ${ }^{17}$ Santa Cruz Institute for Particle Physics and Department of Physics, University of California, Santa Cruz, CA 95064, USA ${ }^{18}$ Department of Physics and Astronomy, University of Utah, Salt Lake City, UT 84112, USA ${ }^{19}$ Department of Physics and Astronomy, University of Alabama, Tuscaloosa, AL 35487, USA ${ }^{20}$ Department of Physics and Astronomy, University of Iowa, Van Allen Hall, Iowa City, IA 52242, USA ${ }^{21}$ Institute of Particle and Cosmos Physics, Universidad Complutense de Madrid, 28040 Madrid, Spain ${ }^{22}$ Department of Physics and Astronomy, University of California, Los Angeles, CA 90095, USA ${ }^{23}$ Institute of Physics and Astronomy, University of Potsdam, 14476 Potsdam-Golm, Germany ${ }^{24}$ Department of Physical Sciences, Munster Technological University, Bishopstown, Cork, T12 P928, Ireland ${ }^{25}$ Department of Physics and Astronomy, Purdue University, West Lafayette, IN 47907, USA 